\title{
La conciencia humana y el emergentismo*
}

Ricardo Braun

Universidad de Lima

Recibido: 11 de junio del 2011 / Aprobado: 2 de julio del 2011

En este ensayo se exploran los conceptos de conciencia y emergentismo, además de otros conceptos asociados al problema del origen y estatus metafísico y epistemológico de la conciencia humana. Se defiende la idea de que de ser viable la tesis del emergentismo como explicación del origen y características de la conciencia, esta tendría que ser un emergentismo fuerte a diferencia de otras alternativas. La teoría emergentista de D. Chalmers es analizada por ser una posición bastante discutida en el mundo académico actual y que representa una visión materialista y no reduccionista de la realidad de la conciencia.

\section{conciencia / emergentismo / psicología no reduccionista}

\section{Human consciousness and emergentism}

This paper explores the concepts of consciousness and emergence, together with other concepts associated to the problem of the origin, and metaphysical and epistemological status of the human conscience. I will defend the thesis that if any emergence theory is to succeed it should be a form of strong emergency as opposed to other alternatives. Due to the high visibility of D. Chalmers's emergence view of consciousness among academics, his theory shall be discussed as a materialist and yet non-reductionist alternative to the reality of consciousness.

\section{consciousness / emergentism /non-reductionist psychology}

\footnotetext{
* Este trabajo es el resultado parcial de la investigación "El emergentismo y la explicación de la conciencia en la filosofía de la psicología contemporánea”, auspiciada por el Instituto de Investigación de la Universidad de Lima al que agradezco por el apoyo y la autorización para publicar este ensayo.

Correo electrónico: rbraun@ulima.edu.pe
} 


\section{INTRODUCCIÓN}

Este artículo tiene por finalidad familiarizar a la comunidad psicológica con el concepto del emergentismo y su importancia en la discusión acerca de la aparición de la conciencia humana. Una de las tareas relevantes de la filosofía contemporánea es el análisis conceptual con miras a aclarar, incorporar o desechar algunos conceptos en diferentes áreas del saber humano. En este caso, el concepto de emergentismo ha cobrado especial importancia en el desarrollo de teorías de la mente que tienen la pretensión de ser materialistas y al mismo tiempo no-reduccionistas. Precisamente la autonomía explicativa de la psicología depende del anclaje metafísico y epistemológico que tengan las entidades, metodología y vocabulario que la disciplina postula y emplea. Y claramente, la psicología contemporánea mantiene estos tres componentes en forma no reducida. El emergentismo, como teoría filosófica de largo recorrido histórico, puede proporcionar los elementos teóricos para conceptualizar mejor el fenómeno de la conciencia humana.

El artículo está divido en tres secciones. En la primera presentaré un análisis del concepto de emergencia y otros conceptos asociados a la discusión del emergentismo, con énfasis en el problema de la causación mental. En la segunda sección presentaré la problemática acerca del origen de la conciencia y las posibles respuestas que se han formulado. En la sección final discutiré la propuesta emergentista de D. Chalmers dada su importancia en la reflexión contemporánea y su posible aplicabilidad en una teoría de lo mental.

\section{EL CONCEPTO DE EMERGENTISMO}

\section{La emergencia en la realidad}

El término emergencia es utilizado en diferentes sentidos, pero nos concentraremos, no tanto en el uso no popular, sino más bien en el vocabulario empleado en el ambiente académico contemporáneo. El concepto de emergentismo es usado generalmente en las doctrinas de orientación no-reduccionistas tanto de la Biología como de la Psicología y otras disciplinas sociales. Veremos, además, su relación con la metafísica y la epistemología psicológica.

Desde una perspectiva metafísica, una postura emergentista concibe que la realidad existe y se presenta en forma plural, de ahí que, en primer lugar, se podría afirmar que el emergentismo es una forma de pluralismo (Bunge, 1977). Sin embargo, el pluralismo que da sentido al emergentismo debe admitir que existen diferentes niveles de organización que son ontológicamente de igual valor.

En segundo lugar, el emergentismo sostiene que en cada nivel superior de organización, aparecen (o emergen) nuevas e irreducibles propiedades que no están presentes en el nivel inferior. 
Estas propiedades serían propiedades "emergentes", que serían predicadas de un todo organizado, y no de sus partes o componentes. Otra forma de presentar la tesis emergentista es sosteniendo que las propiedades de mayor nivel son propiedades de segundo orden, que son el resultado (o emergen) de las propiedades de primer orden.

Desde una perspectiva epistemológica, cualquier doctrina emergentista supone que el conocimiento de las propiedades emergentes no puede ser derivado del conocimiento de las propiedades de las partes que componen el ente emergente, y las leyes que se puedan formular en el nivel emergente no son deducibles de las que se obtienen en los niveles inferiores.

Recientemente el proyecto multinacional "Human Strategies in Complexity. Foundations for a Theory of Evolutionary Systems" (Arshinov \& Fuchs, 2003) ha tratado de sintetizar e identificar características que tienen en común las teorías clásicas y contemporáneas de la emergencia y estas serían:

a) Sinergismo.- La emergencia ocurre como producto de la interacción entre las entidades. La sinergia sería una propiedad de la materia que se manifiesta en la cooperación (sea de partes, elementos, o individuos) y produciendo efectos. La sinergia entre los individuos que interactúan seria la causa de la evolución de la persistencia de los sistemas emergentes. b) Novedad.- En el nivel superior al de las entidades interactuando sinergéticamente, aparecen nuevas cualidades. Estas cualidades no habrían sido previamente observadas y no habrían existido en otro sistema. Esta característica podría ser resumida en el conocido principio que "el todo es mayor que la suma de sus partes".

c) Irreductibilidad.- Las nuevas cualidades producidas no serían reducibles al nivel de las entidades productoras o derivables de este.

d) Impredictibilidad.- La forma de la resultante emergente y el tiempo de la emergencia serían impredecibles.

e) Coherencia/correlación.- Los sistemas complejos que tienen cualidades emergentes tendrían un comportamiento coherente por un cierto periodo de tiempo.

f) Historicidad.- Las cualidades emergentes no están predeterminadas, sino que aparecen como resultado del desarrollo dinámico de los sistemas complejos.

Lo valioso de esta caracterización de cualquier teoría emergente radica en que el trabajo interdisciplinario se nutre del aporte de científicos, filósofos y académicos de diferentes disciplinas, desde las ciencias formales hasta las lingüísticas y semióticas, para quienes las teorías con orientación emergentista podrían convertirse eventualmente en 
nuevos paradigmas para la ciencia actual y en el futuro.

Aun cuando solemos asociar el concepto de emergencia con las ciencias biológicas y humanas, como se mencionó anteriormente, es importante aclarar que las propiedades emergentes pueden ser biológicas. Lo que se requiere para que ocurra un fenómeno emergente en cualquier aspecto de la realidad es la súbita aparición de una nueva cualidad, que pudiera existir temporalmente en un rango desde los microsegundos a los miles de años.

Según Reid (2007), la emergencia biológica e intencional se distingue de la no-biológica por al menos cuatro fenómenos. El primero es la reproducción biológica, que proporciona a los patrones emergentes de complejidad viviente alguna garantía de persistencia. Un segundo fenómeno es el de integridad dinámica o también podría denominarse "automantenimiento". Algunos consideran que esta característica es esencial en los fenómenos emergentes y lo denominan auto-organización (Arshinov \& Fuchs, 2003, p. 6). Los dos primeros fenómenos darían lugar a un tercero, la habilidad para conducir experimentos naturales en la emergencia evolutiva. Finalmente, el cuarto fenómeno que define la emergencia biológica y además intencional, sería también de tipo experimental: los organismos pueden ajustar su integridad dinámica en diferentes entornos ambientales, y así indu- cir cambios fisicoquímicos y biológicos en sí mismos. El incremento de la complejidad en los organismos o la adquisición de nuevas cualidades emergentes pueden ser igualados con el incremento de libertad para dirigirse a actividades específicas. Este cuarto fenómeno, entre otros, podría explicar el paso de la evolución bioquímica que en el caso humano nos llevó desde el comportamiento intencional hasta la aparición del lenguaje en tanto confrontación del cerebro desarrollado con el entorno de supervivencia encontrado (Bickerton, 2009).

Una forma de entender el emergentismo es también describirlo en relación con una posición antagónica en la tradición de la discusión acerca de la realidad mental, a saber, el materialismo reduccionista. El reduccionismo aboga por las explicaciones y la comprensión ontológica en términos y entidades, respectivamente, de las partes constituyentes de algún sistema físico. Una teoría fundamental fisicalista será de mayor profundidad y más incluyente, tendría mayor poder explicativo $\mathrm{y}$ predictivo $\mathrm{y}$, en consecuencia, tendría una comprensión mayor del mundo. La presuposición ontológica implícita es que la realidad física, sea lo que fuere, es la fuente real ontológica de todo lo existente, y cualquier cosa que quiera mantener el estatus de real debiera ser susceptible de ser descrito o construido a partir de aquellos elementos de esta ontología fundamental. 
El dualismo, por su lado, afirma el rol causal eficiente para ciertos fenómenos, tales como las actividades de un alma o mente, que no podría ser derivada de ninguna forma de las propiedades físicas, que no solo no comparte, sino que opone de manera sustancial. Prácticamente, en la filosofía contemporánea, así como en la psicología académica, no hay lugar para el dualismo sustancial cartesiano. Sin embargo, muchas de nuestras discusiones, y en particular, este mismo trabajo, nos muestran que el reduccionismo no nos llega a convencer universalmente y la mayoría de filósofos de orientación analítica adoptan alguna versión del materialismo noreduccionista, sin estar muy satisfechos con la cantidad de contraejemplos que se presentan constantemente. Y en parte, es debido a que no hemos desarrollado una teoría suficientemente consistente del fenómeno de la conciencia. En palabras de T. Nagel, defensor por décadas de la irreductibilidad de lo mental:

Lo que necesitamos es algo que no tenemos: una teoría de los organismos conscientes como sistemas físicos compuestos de elementos químicos y que ocupan un lugar, que tienen también una perspectiva individual en el mundo, y en algunos casos una capacidad para la autoconciencia también... La extraña verdad parece ser que ciertos sistemas físicos complejos biológicamente generados, de los cuales cada uno de nosotros es un ejemplo, tiene ricas propiedades nofísicas (1985, p. 51).
Por ello, cualquier teoría emergentista que pudiera concebirse debe tener condiciones como las señaladas por Crane (2001), que restringen adecuadamente el concepto de emergencia dentro del consenso materialista mencionado. Para Crane, los requisitos serían la distinción y la dependencia. $\mathrm{La}$ distinción significa que las propiedades mentales son distintas de las propiedades físicas y la dependencia, que las propiedades mentales son propiedades de los objetos físicos (p. 208). Las teorías reduccionistas de lo mental, asociadas a la teoría de la identidad tipotipo postulada fundamentalmente por J. J. C. Smart (1962/1991), sostienen que las propiedades mentales son idénticas a las propiedades físicas. La teoría de la identidad sería contraria a la noción de distinción. Las formas del dualismo cartesiano serían una negación al requisito de dependencia.

La noción de emergencia con el requisito de distinción y dependencia significaría que las propiedades emergentes -propiedades mentales para el caso de la filosofía de la mente- serían distintas de las propiedades físicas y, además, que el dualismo de tipo cartesiano sería falso. La idea de que algunas propiedades emergen del sustrato físico significaría en estos requisitos que ellas no podrían ser explicadas ni predichas desde una perspectiva de las ciencias de los sustratos físicos, es decir, de las ciencias físicas. Pero también tiene que haber dependencia con lo físico, y eso 
sería innegable: si destruyéramos las moléculas que componen las neuronas, no podríamos tener neuronas y la actividad cerebral cesaría; el uso de drogas alucinógenas alterna significativamente la actividad mental cognitiva, entre otras.

La conciencia, los eventos mentales y su importancia en el emergentismo

Una de las lecciones que aprendimos de las críticas al positivismo lógico es que algunos problemas son genuinos problemas y las preguntas a esos problemas no son pseudopreguntas. El positivismo había agrupado a las preguntas sobre la conciencia dentro de las pseudo-. Hoy, naturalmente pensamos diferente, y eso porque no podríamos negar algo que, si bien es difícil de conciliar en una teoría evolucionista, fisicalista y lógica, no por eso debe ser descartado. El tema de la conciencia está de nuevo con nosotros, y desde hace ya varias décadas. Su análisis no puede estar ausente de las discusiones sobre el estatus de las entidades y los procesos de la psicología académica y sus cognaticios.

Las teorías emergentes tienen el mérito de enfrentar el problema de la conciencia desde el punto de vista de su origen y también de su existencia como entidad. Sin embargo, no existe una teoría unificada de la conciencia. Son múltiples los sentidos del concepto y por lo tanto, se hace más difícil su evaluación como constructo válido, no solo para su comprensión sino también para su confrontación en el plano empírico. Por poner un ejemplo, en el pasado, la psicología científica estudiaba procesos que no eran conscientes, como los estudios que llevaron a Chomsky a la concepción de una gramática innata que se basaba en el conocimiento inconsciente de las reglas del lenguaje. Otras serán las conclusiones que veremos cuando se aborden más estudios desde el punto de vista de la conciencia, que dado el prejuicio conductista que imperó hasta hace algunas décadas cercanas, fueron descuidados.

Por ello, desarrollaré un breve análisis del concepto de conciencia con miras a proporcionar el análisis conceptual necesario para cualquier discusión posterior.

Según N. Block (1995), el concepto de conciencia (tanto académico como coloquial) es, en el fondo, la combinación de varios conceptos:

La conciencia es un concepto híbrido: hay un número de muy diferentes "conciencias". La conciencia fenomenal es experiencia; el aspecto fenoménico consciente de un estado es lo que significa estar en ese estado. La marca del acceso-conciencia, en contraste, es la disponibilidad para el uso en el razonamiento y la guía racional del habla y la acción (1995, p. 1).

Claro que para Block el resultado de este híbrido es casi desastroso 
para los efectos de la investigación, puesto que lo que se estudie sobre uno puede resultar contradictorio o confuso en otro.

Ciertamente, la posición de Block es incuestionable, pero las funciones denominadas mentales que suelen ser inconscientes, como la mencionada sobre la capacidad lingüística, suelen ser menos problemáticas para asimilarlas a funciones asociadas al modelo computacional, bien sea conexionista $\mathrm{u}$ otras alternativas presentes. Pero de los conceptos mencionados son dos los que deben ser especialmente tomados en cuenta al desarrollar una teoría emergente: la de la conciencia fenoménica y la de la autoconciencia.

La conciencia llamada fenoménica es definida con la celebérrima frase de T. Nagel (1974): “¿Qué es ser como un murciélago?". Esta frase resume la experiencia de ser un sujeto, que experimenta estados cualitativos como ver el color rojo de una manzana, sentir el sabor del chocolate, escuchar la obertura de Parsifal, etcétera, esto que los filósofos llaman los qualia. En otras palabras, ¿cómo es que tenemos esas sensaciones de la manera tan particular como las que experimentamos? Estas sensaciones, además, tienen la propiedad de ser percibidas por nosotros mismos junto a otras simultáneamente, que presumiblemente deben ser procesadas por diferentes partes de nuestro cerebro.

La otra concepción de conciencia hace alusión a nuestra capacidad para dirigir nuestras acciones, controlarlas racionalmente, decimos, a partir de una reflexión que hacemos de nosotros mismos. Nos damos cuenta de que somos agentes, o si se quiere, que somos un Yo, una persona. La autoconciencia supone que uno sabe de sí mismo, sin que esto involucre que uno esté en lo correcto de lo que sabe. Yo me doy cuenta de que estoy con un dolor de estómago aunque más tarde un médico me revele que tengo un cálculo en el riñón. Pero lo que el médico no puede desconformar es mi sensación de darme cuenta de que estoy teniendo el dolor (ahora corregido a una dolencia en el riñón).

Una teoría emergente debe idealmente dar cuenta de cómo es posible que a partir de elementos como partículas subatómicas se generen estos dos tipos de conciencia (y acaso otros más), partiendo de la suposición de que esas partículas no son conscientes en los sentidos explicados. Descartando la existencia de mentes inmateriales, sustancias ideales conscientes y otras suposiciones que tomamos como patentemente falsas, existe evidentemente una brecha explicativa entre los elementos fundamentales o bloques primarios de la realidad que dan evidencia de no ser conscientes y los sujetos como nosotros que tienen la experiencia de ser altamente conscientes. Lo que se requerirá -hay que admitir que no tenemos tales teorías en el momento- es una teoría o teorías que expliquen cómo lo que no es consciente se convierte en consciente. 


\section{Causación emergente}

Toda teoría emergentista tiene que enfrentar el problema de la causación efectiva. Si aparecen nuevas propiedades a partir de propiedades de un nivel inferior, se esperaría que esas nuevas propiedades tengan un real rol causal, en virtud de las nuevas propiedades. Si nos limitamos a los actos conscientes o de la conciencia tendríamos que preguntarnos ¿cómo es posible que la conciencia sea causalmente eficaz? O de forma más precisa, ¿cómo es concebible que los estados conscientes qua conscientes puedan interactuar con estados neuroquímicos qua neuroquímicos? O si se quiere simplificar en un lenguaje ordinario, ¿cómo es posible la causación mental?

Para algunos, la causación mental es una realidad ontológicamente anclada en el mismo nivel superior mental, y en consecuencia, compatible con el discurso emergentista. Esta es la posición que defenderé en este trabajo. Para otros, la conciencia tiene un papel meramente funcional, y en consecuencia, el mecanismo neural es el causante de todo lo que denominamos causación mental (Block, 1995). Otros están convencidos de que, dado nuestro conocimiento científico del mundo, la eficacia causal mental está excluida (Levine, 2001). Finalmente, hay quienes simplemente abandonan cualquier intento de solución y adoptan una posición escéptica ante la posibilidad de explicar la eficacia causal de lo mental (Baker, 1993).
Sin embargo, a pesar de los cuestionamientos que encontramos en la literatura filosófica, desde el punto de vista de nuestra vida cotidiana nos parece que nada podría ser más certero que la eficacia de nuestra conciencia.

Al analizar a diferentes autores que propugnan una teoría emergentista debemos examinar en qué medida se cumple la exigencia de dar una explicación causal a la conciencia y los actos mentales, puesto que no tendría sentido afirmar la existencia de niveles ontológicos autónomos $\mathrm{y}$, al mismo tiempo, negar su eficacia causal o dejarla inerte.

Pero postular la eficacia causal de la conciencia, siendo un gran desafío dentro de una postura fisicalista, permitiría naturalizarla. Las tendencias eliminativistas, por atractivas que parezcan, nos crearían la ilusión de la conciencia causal; sin embargo, nuestra vida y nuestro autoconcepto parten de la experiencia de la conciencia misma. Hay algo perturbador en que por un lado nuestros actos conscientes sean ubicuos en nuestra vida y, si seguimos las conclusiones eliminativistas, resultan ser inexistentes. La naturalización de la conciencia pasa por darle un lugar dentro de la evolución adaptativa. Por azaroso que haya sido el proceso evolutivo, debe de haber habido un fin adaptativo en nuestros ancestros que dio lugar a la aparición de la complejidad estructural y biológica que lleva al fenómeno de la conciencia y de toda nuestra vida mental. Y también deberíamos tener en cuenta en esto último 
la ingente cantidad de recursos energéticos corpóreos que supone el mantenimiento de los fenómenos mentales.

Sin duda, el problema de la causación surge en el contexto de cualquier teoría emergentista. En efecto, uno de los problemas que de modo permanente ronda al emergentismo es la posibilidad de que la realidad emergente de la que se predica resulte ser meramente un epifenómeno sin un rol causal eficiente o, peor aún, ficticio. Veamos.

\section{Epifenomenalismo}

El epifenomenalismo es la postura que sostiene que los fenómenos mentales son causados por eventos físicos en el cerebro, pero no tienen efectos sobre ningún evento físico, incluido el mismo cerebro. Entonces, la conducta es causada por músculos que se contraen al recibir impulsos nerviosos, y los impulsos nerviosos generados por la entrada de otras neuronas o de órganos sensoriales. La conclusión a que se llega con el epifenomenalismo es que los eventos mentales son ineficaces, como lo son las experiencias cualitativamente mentales. En todo caso, el epifenomenalismo es tomado hoy como una visión crítica contra el no-reduccionismo, puesto que cualquier fenómeno que no sea físicamente describible y anclado ontológicamente en las entidades físicas, no tiene poder causal dentro del concepto de cierre causal del mundo. Si el epifenomenalismo fuera correcto, entonces, los procesos emergentes no serían causalmente relevantes, sino que habría que buscar su base física como explicación de su poder causal. En otras palabras, no es lo emergente lo que causa algo sino puramente el arreglo físico correspondiente.

Lo que motiva el epifenomenalismo es la suposición de que todos los eventos físicos tienen las causas necesarias y suficientes, y estos pertenecen a un único nivel de eventos físicos. Si los eventos biológicos, mentales o sociales fueran algo diferente a un evento físico, entonces cualquier contribución propia sería una violación de las leyes físicas y al principio de cierre causal universal. Podemos ver claramente que es una tesis opuesta al pluralismo ontológico.

Es interesante mencionar que el problema del epifenomenalismo surge sobre todo en la teoría de la mente y no, por ejemplo, en las teorías de la biología, o aun en la química orgánica, puesto que si el mundo fuera explicado y dependiera ontológicamente de las micropartículas, en la práctica todo sería un producto epifenomenalista. La importancia se puede aclarar aún más. Si se demostrara que las propiedades mentales son causalmente impotentes en comparación con las propiedades físicas, ¿cuál sería la razón para mantener una ontología que es inerte o inactiva? Se podría aducir que son como los objetos abstractos que forman parte de nuestro discurso coloquial y científico. Pero realmente los objetos abstractos no son entidades (relata) que tienen un rol causal, no es- 
peramos que lo tengan. Si mantenemos nuestras categorías mentales es porque también confiamos que ellas tienen que ver con los cambios causales efectivos en el mundo: creemos que nuestros deseos, como nuestra voluntad sí tienen causación eficiente en nuestros asuntos. Como dice P. Humphreys:

[...] si el argumento de la exclusión [el verdadero trabajo causal es debido a las propiedades físicas] entonces el raciocinio, los qualia y las esperanzas y temores de la humanidad son simplemente humo en el fuego de los procesos cerebrales $(1997$, p. 2).

T. H. Huxley fue uno de los primeros que lo utilizó en el contexto de lo mental a mediados del siglo XIX, comparando los eventos mentales con el silbato de la máquina de vapor que no contribuye en nada al funcionamiento mecánico de la locomotora. William James, a fines de dicho siglo, rechazó esta postura utilizando un argumento basado en la selección natural. Decía que si los placeres y los dolores no tuvieran efectos, no habría razón alguna para nuestra aversión a esas sensaciones que son causadas por actividades que consideramos importantes en nuestra vida. Entonces no se explicaría por qué tenemos una valoración afectiva a las sensaciones y a la utilidad de las actividades que las producen (Robinson, 2007).

El problema del epifenomenalismo no es otro que el problema denunciado por el materialismo eliminativista.
Podemos ver que tanto los eliminativistas como los críticos de la posición emergentista de una realidad ontológica nueva comparten las siguientes convicciones acerca de lo mental:

1. Los conceptos mentales son equivalentes a conceptos vacíos puesto que sus términos se refieren a algo que no existe.

2. Existen estados mentales, pero ellos son simplemente estados cerebrales.

De acuerdo con el primero (1), el eliminativismo es un eco de la eliminación de muchos conceptos, con sus respectivos términos, que resultaron de hacer referencia a cosas inexistentes, como por ejemplo el flogisto, los demonios, las esferas de cristal y conceptos parecidos. Según el segundo (2), hay un reconocimiento de la existencia de los estados mentales -en oposición al conductismo lógico y metodológico original- pero requiere una reconceptualización teniendo como marco conceptual a las neurociencias. Es decir, no es que los estados mentales no existan, sino que están inadecuadamente descritos en el lenguaje de origen popular mentalista y debieran ser traducidos o reemplazados por conceptos neurológicos en una futura ciencia de la conducta humana.

Smart, que fue mencionado anteriormente, sostiene lo siguiente:

Soy de la opinión que la ciencia nos está proporcionando más y más un punto de vista por el cual los orga- 
nismos pueden ser vistos como mecanismos físico-químicos: me parece que aún el comportamiento del mismo hombre será explicable algún día en términos mecanicistas. Parece haber, en lo que concierne a la ciencia, nada en el mundo sino un creciente arreglo complejo de los constituyentes físicos (1962/1991, p. 169).

Sin embargo, el temperamento de Smart no lo llevaba a postular la necesaria eliminación en el momento del vocabulario con contenido ontológico mental, sino como una propuesta a futuro. P. M. Churchland, sin embargo, sí es de la convicción de que en tanto más pronto nos deshagamos de toda la ontología mental, tanto mejor. Su eliminativismo se expresa así:

[E]s la tesis que nuestra concepción común de los fenómenos psicológicos constituye una teoría radicalmente falsa, una teoría tan fundamentalmente defectuosa que tanto los principios y la ontología de tal teoría eventualmente serán desplazadas, en vez de suavemente reducidas, por una neurociencia completa. Nuestra comprensión común y aun nuestra introspección será entonces reconstituida dentro del marco conceptual de una neurociencia completa, una teoría que podemos esperar sea mucho más poderosa que la psicología de sentido común que desplaza, y sustancialmente más integrada dentro de la ciencia física en general (1981/1991, p. 601).
Por lo tanto, el epifenomenalismo se presenta como una objeción a la emergencia también porque negaría que los eventos emergentes sean una realidad ontológica como serían otras realidades que sí participan causalmente en el mundo. Si no se supone que las propiedades emergentes tienen efectos, entonces bien podrían no ser propiedades reales sino inertes, o epifenoménicas. Y mucho de lo que se dice en el emergentismo podría tener la objeción de ser epifenoménica. Como dice Flanagan: "La sospecha epifenomenalista es extraordinariamente difícil de disipar" (1992, p. 133). El materialista, reduccionista o no, es pues, contrario al emergentismo, como lo señala adecuadamente Horgan:

Una posición materialista debería aseverar, contrariamente al emergentismo (i) que la física es causalmente completa (es decir, las fuerzas causales fundamentales son fuerzas físicas, y las leyes de la física no pueden ser nunca violadas); y (ii) que cualquier hecho metafísicamente básico o leyes -cualquier explicador inexplicado, por decirlo así- es un hecho o leyes dentro de la misma física (1993, p. 560).

Se puede ver, entonces, que la tensión es clara entre el emergentismo y el materialismo. El emergentismo sostiene que los eventos mentales tienen poderes causales que no son explicables en los términos ni ontología de los poderes causales de sus substratos físicos. Pero 
el materialismo postula que los poderes causales de lo mental son explicables en términos de las propiedades y leyes físicas subyacentes. Por eso, para los materialistas es importante negar que las propiedades mentales sean propiedades emergentes, y si lo fueran, posiblemente serían microrreducibles.

\section{Superveniencia}

El concepto de superveniencia también es un concepto central en la discusión de la causación mental y, en consecuencia, de cualquier teoría emergentista. Como muchos conceptos que son de uso casi exclusivo de la comunidad filosófica, el de superveniencia es uno que ha resurgido en la discusión académica reciente en el problema de lo mental, particularmente impulsado por J. Kim (1993).

La superveniencia no es propiamente una teoría sino un concepto aplicado a las relaciones entre dos conjuntos de propiedades: las propiedades supervenientes y las propiedades de su base. Kim define así el concepto:

Las propiedades mentales supervienen sobre las propiedades físicas, en que necesariamente, para cualquier propiedad mental $\mathrm{M}$, si algo tiene $\mathrm{M}$ en un tiempo $t$, existe una propiedad física base (o subveniente) $P$, tal que tiene $\mathrm{P}$ en $\mathrm{t}$, y necesariamente cualquier cosa que tiene $\mathrm{P}$ en un tiempo tiene $\mathrm{M}$ en ese tiempo (2000, p. 9).
Supongamos que una persona desea tomar un helado de chocolate. Ese deseo debe ser una instancia de una propiedad física (posiblemente una propiedad neurológica) tal que cuando cualquiera que esté en esa instancia, y tiene aquella propiedad física, habrá de experimentar el deseo de tomar un helado de chocolate. Entonces, cada propiedad mental posee una base física que garantiza esa instanciación. Además, sin esa base física no puede darse la propiedad mental.

Los filósofos han empleado este concepto para imaginar mundos posibles como el de los gemelos de Putnam porque pone la siguiente restricción de realización física: "Cualesquiera dos cosas que son duplicados físicos exactos, necesariamente son también duplicados psicológicos -esto es-, duplicados físicos son duplicados tout court (Kim, 2000, p. 10).

Aparentemente, uno podría considerar que la noción de superveniencia haría más viable la identidad mente-cerebro, o descartar la emergencia, ya que la base física es lo que determinaría los fenómenos mentales. Además, podría sospecharse una acusación epifenomenalista acerca de los fenómenos mentales. Sin embargo, esta noción más bien es consistente con una tesis monista de la realidad, pero no es necesariamente reduccionista, puesto que la realización física de cualquier objeto complejo puede ser variada o múltiple. Lo único que restringe es la posibilidad de que algu- 
na cosa emergente o no tenga el mismo arreglo físico y no tenga las mismas propiedades emergentes o no. Pero la superveniencia es perfectamente compatible con el emergentismo, y aun más, creo que lo fortalece.

En primer lugar, la superveniencia significa que un nivel de los fenómenos depende de otro nivel (por ejemplo, el mental del biológico y este del químico), pero al mismo tiempo no es reducible al último, de ahí que se hable de dos conjuntos de propiedades, de lo contrario se hablaría de uno solo. Utilizando el ejemplo que dio origen al concepto de superveniencia en la moral, una cosa es ser "San Francisco" y otra es ser "bueno". Es importante diferenciar aquí lo que constituye una sutileza importante en el concepto de superveniencia. La superveniencia puede ser fuerte o débil. Será fuerte si cualquier diferencia en el nivel superveniente; así, tener un deseo diferente al de comer helado de chocolate resultaría en alguna diferencia en los sistemas subvenientes (por ejemplo, el arreglo específico del estado del cerebro o del sistema nervioso central). Pero comprometerse a una superveniencia fuerte sería asumir tácitamente que lo que proporciona la explicación real del fenómeno en cuestión sería el nivel subveniente y no el superveniente, como la teoría de la emergencia reclamaría.

En segundo lugar, el concepto de superveniencia puede ir acorde con la teoría de la evolución, en tanto se considere que la base física es necesaria para la aparición de fenómenos emergentes, pero la base física no es lo mismo que los fenómenos emergentes. En otras palabras, se pueden utilizar algunos principios y leyes de la física para describir las condiciones para la aparición de fenómenos, que debido a su arreglo particular da lugar a propiedades novedosas. Sin embargo, no son las leyes de la física las que describirán completamente esos fenómenos. La sensación de ver el color rojo no es la reflexión en un lector óptico de una longitud de onda de 7.700 ángstroms. Eso sería lo que algunos reduccionistas considerarían una muy buena física, pero una ¡muy mala biología!

\section{Causación descendente}

Como hemos visto, la causación eficaz es un problema que acosa al emergentismo, por ello surge la necesidad de postular el concepto de causación descendente, que posiblemente es el concepto más importante que define a la ontología y los procesos emergentes. Según algunos sería la característica más importante del emergentismo de tipo fuerte (Clayton, 2004, p. 49).

El concepto fue introducido por D. Campbell en 1972 y publicado en español en 1974 (Campbell, 1974). Aun cuando la formación de Campbell fue en Psicología, su ensayo estaba más centrado en la Biología. Y es que, como muchos, se encuentra en el caso de la Biología un paso intermedio en los procesos emergentes que alcanzan una ma- 
yor complejidad con la aparición de la conciencia y la conducta humanas.

El objetivo de Campbell era dar cuenta del comportamiento de sistemas biológicos organizados pero dispuestos jerárquicamente. Así, tendríamos en orden ascendente: la molécula, la célula, el tejido, el órgano, el organismo, la población en reproducción, la especie, el sistema social, hasta el ecosistema. Pero sostiene que esas no son realidades explicables desde el punto de sus componentes y menos concebidos como meros "agregados" sino más bien

[...] como realidades verdaderas que como conveniencias arbitrarias de clasificación, organizando cada uno de los órdenes superiores las unidades reales del nivel inferior (1974, p. 237).

Es decir, son realidades en todo el sentido de independencia ontológica. Para los reduccionistas -y, curiosamente, Campbell se incluye entre ellos- las realidades jerárquicamente superiores se restringen por las leyes que gobiernan el comportamiento de las realidades inferiores, incluidos los niveles de la física subatómica (1974, p. 237). Este sería considerado un principio reduccionista de dos. Pero para Campbell no es suficiente, porque no se explicaría adecuadamente el fenómeno de la selección natural. Por ello, introduce dos principios adicionales:

- El principio emergentista: "La evolución biológica en su exploración sinuosa de segmentos del universo tropieza con leyes, que operan como sistemas selectivos, que no están descritas por las leyes de la Física y de la Química inorgánica, y que no serán descritas por los sustitutos futuros de las aproximaciones actuales de la Física y de la Química inorgánica" (p. 237).

- El principio de la causación descendente: "Cuando la selección natural actúa mediante la vida y la muerte en un nivel superior de organización, las leyes del sistema selectivo de nivel superior, determinan, en parte, la distribución de los hechos y sustancias de nivel inferior" (p.237).

La causación descendente también puede ser entendida (y es la formulación más conocida de Campbell), como una reformulación del primer principio:

Todos los procesos en los niveles inferiores de una jerarquía son restringidos por las leyes de los niveles superiores y actúan en conformidad con ellas (Campbell 1974, pp. 237-238).

Entonces, para Campbell la causación ascendente es condición necesaria en la descripción y explicación del fenómeno biológico, pero no es condición suficiente, es solo parcial. En este sentido, además de una explicación en términos bioquímicos, se requeriría una explicación causal descendente para complementarla. Se pregunta, ¿cómo se podría explicar el hecho de que las 
mandíbulas de una termita o de una hormiga obrera pudieran estar tan bien diseñadas como para hacer el trabajo de roer madera y de cargar semillas? Ciertamente, las leyes de la palanca son una parte de la explicación del organismo, pero se requiere del principio de "propósito organísmico" para explicar la distribución particular de las proteínas en la mandíbula, es decir, las plantillas de ADN que guían su producción (Campbell, 1974, pp. 238-239).

La causación de otros elementos del organismo también debe ser incorporada, como la práctica social y los elementos del entorno que influyen en el diseño. Dice Campbell:

Las mandíbulas de la hormiga soldado y la distribución de las proteínas en ellas requieren para su explicación ciertas leyes de la Sociología centradas sobre la organización social en la que existe una división del trabajo (1974, p. 239).

La causación descendente, por lo tanto, no estaría interfiriendo con principios y regularidades biológicas ni tampoco con las leyes de la física. En otras palabras, dentro de una concepción fisicalista, la realidad de la vida trae nuevas formas de interacciones causales en el mundo, pero ellas son, a su vez, consistentes con la física, pero no explicables ni predecibles por la física. Por poner un ejemplo, las leyes de la física no pueden explicar y predecir la aparición de ratas, pero las ratas y su comportamiento no violan las leyes de la física.

Ahora bien, por más intuitivamente correcto que aparezca el concepto de causación descendente, no ha sido inmune a una serie de críticas, particularmente cuando se utiliza el concepto en el análisis del fenómeno de la conciencia. Para los objetores, las actividades de la conciencia, y en general de lo que llamamos eventos mentales, se explican necesaria y suficientemente por la causación ascendente: las influencias causales ocurren exclusivamente desde las partes constituyentes al todo constituido. Uno podría creer que el contenido de un pensamiento fue lo que causó una determinada acción; sin embargo, de hecho las fuerzas causales operativas son los eventos microfísicos, que en nuestros cerebros tienen la forma de eventos electroquímicos.

Se podría afirmar la causación descendente desde otra perspectiva, la dualista. Los dualistas afirmarían que una entidad o entidades que son ontológica y radicalmente distintas a las causas físicas son las responsables de la influencia causal en nuestros cuerpos. Pero esta posición, poco convincente en nuestros días por razones empíricas, también tiene problemas en el nivel conceptual. Por ejemplo, cuando se afirma que existen cosas ontológicamente distintas, existe una tremenda ambigüedad por el sentido de cualitativamente distintas, o en el caso histórico cartesiano, de naturalezas distintas. Pero esas distinciones 
podrían aplicarse al mundo biológico (sin incluir a los humanos conscientes) sin ninguna sorpresa. Por ejemplo, un conejo y un electrón parecen compartir poco en sentido ontológico; sin embargo, no tendríamos que inclinarnos al dualismo para explicar que los movimientos del conejo pueden afectar a los electrones que lo componen.

Para los comportamientos de los seres vivos elementales, como los paramecios, es posible que una explicación en los niveles epistemológico y ontológico basada en la bioquímica pareciera ser suficiente, aun así algunos no compartirían como suficiente una explicación bioquímica. Pero cuando extrapolamos la bioquímica a la acción humana la brecha se hace inalcanzable, y las explicaciones y ontología que suponen una causación ascendente parecen insuficientes. Por ejemplo, en las explicaciones de los procesos psicológicos o históricos la causación ascendente nos conduciría a versiones que por momentos parecen cómicas. Supongamos que quisiéramos explicar las causas de la Segunda Guerra Mundial a partir de los eventos del fin de la Primera Guerra Mundial. ¿Qué vocabulario y qué entidades debieran reemplazar a las que usualmente utilizamos en la historiografía para dar cuenta de lo que algunos llamaron la "conspiración" de los veteranos de la Primera Guerra Mundial? ¿Es que la física fundamental -partículas subatómicas- conspiraron (o algún fenómeno que asociamos con la conspiración) para causar la Segunda Guerra? ¿No parece más razonable acudir a nuestras tradicionales concepciones sociológicas/históricas como entes responsables de la causación de estos conocidos eventos?

Por supuesto, el hecho de que algo parezca cómico o antiintuitivo no lo hace, en principio, falso como sistema explicativo. Sin embargo, las explicaciones poderosamente antiintuitivas, sobre todo cuando tratan acerca de nosotros mismos, nuestras acciones, voliciones o intenciones pueden ser sospechosas de no ser adecuadas, y por ello la concepción de causación descendente puede ser poderosamente explicativa en el análisis causal de, al menos, un grupo de fenómenos de la compleja realidad.

\section{Tipos de emergencia}

Para finalizar el análisis conceptual sobre el emergentismo, veamos algunos tipos de teorías emergentistas, puesto que, como toda teoría, la variedad de presupuestos y postulados de cada teoría determinará sus compromisos -ontológicos y epistemológicos- en el esfuerzo por construir una teoría aceptable de la conciencia. Además, en la última sección analizaremos una conocida postura emergentista, y por ello, sería importante visualizarla dentro de la tipología que a continuación describo.

\section{Emergentismo fuerte}

El emergentismo fuerte sostiene que un fenómeno de nivel jerárquicamente alto 
es fuertemente emergente con respecto a un dominio de nivel inferior si el fenómeno de mayor nivel surge del dominio inferior, pero las verdades que conciernen al fenómeno no pueden ser deducibles, ni en principio, de las verdades del nivel inferior. Se podría decir que la evolución del cosmos produce niveles nuevos, ontológicamente distintos, que se caracterizan por sus propias leyes o regularidades, y sus fuerzas causales. Esta concepción es la más usual en la discusión filosófica y caracterizó a los llamados "emergentistas británicos" de principios del siglo XX.

El emergentismo fuerte tiene un compromiso ontológico más radical que el débil, puesto que considera que las características emergentes ontológicamente son características de los sistemas o de los todos que poseen capacidades causales que no son reducibles a ninguna de las capacidades causales de las partes.

\section{Emergentismo débil}

El emergentismo débil sostiene que un fenómeno de nivel jerárquicamente alto es débilmente emergente con respecto a un dominio de nivel inferior si el fenómeno de mayor nivel surge del dominio inferior, pero las verdades concernientes al fenómeno son inesperadas dados los principios que gobiernan el dominio del nivel inferior. Los emergentistas débiles afirman que, conforme aparecen nuevos patrones y características, los procesos causales pertenecen al ámbito de la física.

El emergentista débil está de acuerdo con el fuerte en que la evolución produce nuevas estructuras que, como un todo emergente, determina a sus partes. Pero, a diferencia del fuerte, admite que la imposibilidad de reconocer la estructura de los componentes que dan origen a la emergencia del nuevo todo, se debe a nuestra ignorancia. En otras palabras, más que una posición ontológica es una posición epistemológica. Las características novedosas lo serían solo en el nivel epistémico.

Como se puede inferir, la emergencia fuerte tiene consecuencias mucho más radicales que la débil, puesto que si se quiere entender los fenómenos de los niveles más altos se requerirá el conocimiento de nuevas leyes y principios fundamentales que no se conocen en la física.

Puesta así la diferenciación, pareciera que la posición débil es insostenible y sorprendería porqué debiera llamarse emergentismo después de todo, porque es una posición perfectamente compatible con el fisicalismo reduccionista. La cuestión es más de tiempo que de ontología. Conforme se vaya descubriendo más el mundo, estaremos en capacidad para asimilar los eventos de nivel superior en los del nivel inferior, tarea que ya se había empezado en tantos ejemplos conocidos, desde el siglo XIX hasta nuestros días, en el caso de la biolo- 
gía molecular, por mencionar el modelo reduccionista más impresionante.

\section{De la Biología a la concienCia}

No es solo una estrategia explicativa, y -espero- persuasiva, el encontrar una transición, un continuumm desde la Biología hacia la conciencia humana, sino que desde una perspectiva evolucionista, no tiene sentido hablar de la conciencia sin tomar en cuenta la historia evolutiva que produjo los cerebros. Para el dualista no es necesario siquiera enfrentar la pregunta de este tránsito porque casi por milagro aparecen propiedades que no tienen nada en común con el resto del orden natural. Aunque parezca insólito, podría decirse algo análogo de los fisicalistas reduccionistas, puesto que el hecho de que haya cerebros biológicos o no es un detalle contingente, ya que al final todo puede subsumirse a leyes microfísicas, incluyendo las funciones cerebrales. Sin embargo, en una propuesta emergentista, se tiene que estudiar la naturaleza de otras estructuras emergentes como parte de la historia evolutiva. Por ello, las teorías emergentistas que vamos a ver a continuación han sido escogidas por distanciarse tanto de las posturas dualistas como de las reduccionistas, pero fundamentalmente porque no descuida la importante cuestión del tránsito evolutivo.

Si queremos entender los fenómenos conscientes no podemos dejar de lado la comprensión de los distintos niveles de la estructura del mundo. En este sentido, la aparición de la conciencia y de los otros fenómenos mentales es un caso más de emergencia en el que los sistemas naturales dieron origen a estructuras y propiedades novedosas. Pero la conciencia es un fenómeno emergente; en consecuencia, no se puede defender una teoría emergente del cerebro sin endosar explícitamente una versión fuerte de la emergencia. La versión débil no hará sino convertir eventualmente la emergencia en un epifenómeno sin ningún interés causal real.

De forma preliminar sería importante reflexionar acerca de los diferentes niveles de complejidad que aparecen como producto de procesos emergentes. Podríamos resumir al menos tres niveles de emergencia natural siguiendo la propuesta de Clayton (2004). Subrayo la idea de resumen puesto que estos niveles podrían ser subdivididos y no todos los biólogos estarían de acuerdo en una división en tres niveles. ${ }^{1}$ El primero sería la aparición de la vida a partir de la materia inorgánica. Actualmente se acepta como una alta probabilidad la emergencia de la vida, dada la presencia de elementos pesados que han debido intervenir, ya sea como componentes o como catalizadores en la combinación que permitió la existencia de este nivel. Sin embargo, reconocemos al mismo tiempo que las fronteras entre lo no-

1 Por ejemplo, Emmeche, Køppe \& Stjernfelt (1997) distinguen hasta cuatro niveles. 
viviente y lo viviente no son tan claras como se creyó cincuenta años atrás. En efecto, hoy en día objetos como los virus tienen características que los asemejan más a cristales que a células epiteliales. ${ }^{2}$ Pero si hay algo que comparten como patrones distintivos los entes vivientes -virus incluidos-son el crecimiento y desarrollo, la homeostasis, la reproducción y el intercambio energético con el entorno ambiental. Además, los seres vivientes son susceptibles de mutaciones genéticas y ambientales siguiendo los procesos de adaptación evolutiva.

En un segundo nivel encontraríamos el monitoreo externo e interno del organismo. En el sentido biológico es una forma elemental de autoconciencia, puesto que involucra no solo darse cuenta del ambiente exterior, que no es lo mismo que la percepción, y además de los propios estados internos del organismo, y la modificación de los comportamientos como resultado de la conciencia.

En un tercer nivel, que lo relacionamos con el concepto de conciencia humana explicado en la sección anterior, encontramos la autoconciencia reflexiva. En este nivel el organismo, como diría Locke (1690/2008), percibe que está percibiendo. En este nivel encontramos fenómenos como el de darnos cuenta de que estamos pensando, que conocemos nuestros propios pensamientos o que conocemos lo que es estar experimen-

$\overline{2}$ Véanse las ilustraciones en Harrison (1989). tando algunos qualia. Esta clasificación de Clayton tiene el valor de que las experiencias del tercer nivel están ancladas en el desarrollo biológico evolutivo, al menos desde un estadio preconsciente, y el conocimiento consciente estaría construido posteriormente partiendo con el anterior.

\section{El origen de la conciencia}

Nuestras reflexiones anteriores nos 1levan al problema central de este trabajo: las teorías sobre el origen del fenómeno de la conciencia. Pero deseo enfocar el problema del origen desde una perspectiva ahistórica, es decir, concentrando la discusión no tanto en lo ontogenético y filogenético, sino más bien en la relación entre la conciencia y el cerebro humano. No hay un sabor no-naturalista en este enfoque, puesto que sin investigar su origen histórico, sin embargo podemos tratar de enfrentar la difícil pregunta acerca de dicha relación: ¿cuál es la relación naturalista entre la conciencia y el cerebro?

Se diría que la pregunta es ahistórica en el sentido de que nos podemos preguntar sobre cómo los estados mentales en este momento (el estado consciente, por ejemplo) se relacionan con los estados cerebrales. Esta pregunta no es otra que la ancestral pregunta del problema mente-cuerpo o mente-cerebro. Sin embargo, no todas las respuestas al problema han sido las mismas, aun cuando hayan tenido una misma motivación naturalista. Veamos las más re- 
presentativas con un claro componente naturalista.

En los últimos cincuenta años se han dado, en general, tres tipos de respuestas a la pregunta por el origen de los fenómenos de la conciencia. Históricamente la primera fue la identificación de los estados mentales con los estados cerebrales, o la teoría de la identidad mente-cerebro (Smart, 1962/1991; Amstrong, 1970/1980).

La segunda, que nació con la crítica de Putnam a la teoría de la identidad y resultó en el funcionalismo (Putnam, 1980). Cualquier versión del funcionalismo tiene en común la aseveración de que estar en un determinado estado consciente es estar (realizar) un rol funcional específico relativo a algún sistema. La relación entre el cerebro y la mente es una de tipo funcional, es decir, es una especie de rol residente en el cerebro. La analogía clásica ha sido que cerebro es a mente como hardware es a software. Lo que importa no es tanto lo que sean las mentes sino lo que hacen. Como el funcionalismo es heredero de la teoría de la identidad, el funcionalista podría identificar los estados mentales con estados cerebrales, en tanto se les tome en la relación funcional, pero bien podrían ser otros realizadores físicos, como las computadoras eventualmente.

Una tercera posibilidad es que la relación entre la conciencia y el cerebro sea una relación emergente, es decir, que los estados conscientes o propiedades son estados o propiedades de mayor nivel $\mathrm{u}$ orden de los estados cerebrales y sus propiedades. De acuerdo con esta visión, los estados mentales dependen del cerebro o de algún estado físico, pero no son iguales a ellos. La emergencia también ha sido entendida con el concepto filosófico de superveniencia, sosteniendo que los estados conscientes supervienen en los estados cerebrales (Kim, 1993).

\section{La teoría emergentista de D. Chalmers}

En esta sección quiero concentrarme en la discusión de la propuesta de David Chalmers acerca del origen de la conciencia, dado que su propuesta ha sido objeto de numerosos e influyentes análisis críticos en los últimos años.

La preocupación de Chalmers por el fenómeno de la conciencia lo lleva a escribir su obra más ambiciosa: $L a$ mente consciente: En búsqueda de una teoría fundamental (1996), en la que se argumenta que las explicaciones reduccionistas de la conciencia son imposibles y que la única forma de enfrentar el problema de la conciencia es abandonar un marco de referencia puramente materialista. Parte de este trabajo pretende desarrollar una teoría de la conciencia en que se puedan relacionar las leyes físicas con la experiencia fenoménica de una manera sistemática. En un sentido estricto no se podría considerar la obra de Chalmers como una nítida defensa del emergentismo, sin embargo, otras obras de Chalmers, y aun esta misma, son maneras de defender, por un lado, 
la autonomía ontológica de la conciencia y su correspondiente explicación no reduccionista, y por el otro, buscar una visión naturalista a la experiencia fenoménica. Estos objetivos podemos enmarcarlos en una visión emergentista, como veremos.

Toda teoría emergentista anclada en una teoría naturalista debe comprender la conciencia como un fenómeno emergente en el mundo natural, incluidos los actos que consideramos mentales como la volición, las actitudes proposicionales, el pensamiento. Además, estos actos deben tener causación efectiva y requieren una explicación naturalista. Ninguna teoría de la conciencia puede desconocer estos fenómenos y es precisamente lo que Chalmers aborda en una obra anterior a la mencionada (Chalmers, 1995). Al problema de dar respuesta al origen de estos fenómenos lo denomina "el problema difícil", y, sin embargo, todas las respuestas que se han dado al problema de la conciencia son las respuestas a los problemas "fáciles". Anota que los problemas fáciles son los siguientes (1995, pp. 200-201):

- Habilidad para discriminar, categorizar, y reaccionar a los estímulos del ambiente.

- La integración de la información a través de un sistema cognitivo.

- La reportabilidad de los estados mentales.

- La habilidad de un sistema para acceder a sus propios estados internos.

- El foco de atención.
- El control voluntario de la conducta.

- La diferencia entre la vigilia y el sueño.

Todos estos fenómenos están asociados a la conciencia. Claro que suena extraño que los considere problemas fáciles y que, además, existan respuestas para su explicación. Sería muy estimulante saber, por ejemplo, si existen respuestas convincentes a comportamientos como el control deliberado de la conducta desde un punto de vista neurofisiológico. Pero según Chalmers, por más difíciles que nos puedan parecer estos problemas no son nada comparados con los que siguen:

El verdadero problema de la conciencia es el problema de la experiencia. Cuando pensamos y percibimos, hay un zumbido de procesamiento de información, pero también hay un aspecto subjetivo... El aspecto subjetivo es la experiencia. Cuando vemos, por ejemplo, experimentamos sensaciones visuales: la cualidad sentida de lo rojo, la experiencia de lo oscuro y de la luz, la cualidad de la profundidad en un campo visual. Otras experiencias siguen a la percepción en diferentes modalidades: el sonido de un clarinete, el olor de las polillas. Luego están las sensaciones corporales, desde los dolores a los orgasmos; las imágenes mentales que se evocan internamente; la cualidad sentida de la emoción, y la experiencia de una corriente de pensamiento consciente. Lo que une a todos estos estados es 
que hay algo que significa estar en ellos. Todos ellos son estados de la experiencia (1995, pp. 201-202).

Esta cita nos revela la problemática de enfrentar las explicaciones en términos de funciones o estructuras, puesto que se podría conocer la estructura o la función de alguna experiencia y, sin embargo, no saber lo que es experimentarla. Lo que lleva a Chalmers a concluir, correctamente creo, que las explicaciones de la Biología o de la Neurología en particular, no parecen ser suficientes. La Neurología puede entender las estructuras y funciones de las células, los órganos, las regiones cerebrales y hasta los organismos. Las neurociencias nos pueden proporcionar teorías que logran tener correlatos neurales de la experiencia consciente. Pero estas explicaciones no parecen agotar la pregunta acerca de lo que son estas experiencias. Algunos teóricos desean añadir algunos ingredientes más. Y es aquí donde el terreno se hace más arenoso y los peligros (como el epifenomenalismo) empiezan a incrementarse.

Chalmers tiene algunas propuestas. Una década después de sus anteriores publicaciones (1995; 1996), Chalmers reconoce que su posición es muy cercana al emergentismo fuerte asociado con la causación descendente, combinación que se presenta como robusta, puesto que un emergentismo débil sería incoherente con la causación descendente (si se puede reducir todo finalmente a la física y microfísica, ¿para qué molestarse en explicar la causación mental?). Dice Chalmers:

¿Existen fenómenos emergentes fuertes? Mi visión es que la respuesta a esa pregunta es sí. Creo que hay exactamente un caso de un fenómeno emergente fuerte, y es el fenómeno de la conciencia. Podemos decir que el sistema es consciente cuando hay algo es que es ser ese sistema; esto es, cuando hay algo que siente como la perspectiva del propio sistema. Es un hecho clave de la naturaleza que ésta contiene sistemas conscientes; yo soy uno así. Y hay una razón para creer que los hechos acerca de la conciencia no son deducibles de ningún número de hechos físicos (2006, p. 248).

Entonces, ¿cómo se relacionan los eventos conscientes con los eventos cerebrales? Chalmers recurre al concepto de superveniencia: "La conciencia superviene en el dominio físico del mundo, parece ser un hecho nómico que la duplicación de los estados físicos duplicará la conciencia; pero en otros mundos con diferentes leyes, un sistema físico idéntico a mí podría no tener conciencia en absoluto" (2006, p. 248). Podemos ver que, en este caso, Chalmers se compromete con la tesis de la superveniencia, pero solo la superveniencia local, no la global. ${ }^{3}$

3 Para la diferencia véase Kim (1993, pp. 53-91). 
La explicación última del origen de la conciencia está bajo el paraguas de sus tres principios desarrollados en Chalmers (1996):

- El principio de coherencia estructural.- Este principio vincula la estructura de la conciencia y la estructura de la subjetividad. Los contenidos de la subjetividad deben ser entendidos como los contenidos informacionales que son accesibles a los sistemas centrales de una manera generalizada para el control global directo. Los contenidos, añade Chalmers, podrían ser expresados en un sistema de lenguaje y ser potencialmente reportables.

- El principio de invarianza organizacional.- Este principio establece que cualquiera de dos sistemas con la misma organización funcional al mínimo detalle deberán tener las mismas experiencias cualitativas. Lo que importaría en este principio es que la emergencia de la experiencia no está determinada por una realización física específica sino por el patrón abstracto de la interacción causal entre sus componentes.

- La teoría de la información de doble aspecto.- Los dos principios anteriores son considerados por Chalmers como no-básicos. Pero la teoría de la información de doble aspecto parte de la observación de que hay un isomorfismo entre la información físicamente enraizada y ciertos espacios de información fenoménica.
La información, entonces, tiene dos aspectos, uno físico y otro fenoménico. Para Chalmers esa diferencia de aspectos puede subyacer y explicar la emergencia de la experiencia a partir de lo físico. La experiencia surge en virtud de ser un aspecto de la información, cuando el otro aspecto se encuentra incorporado en el procesamiento físico. Este principio es el que permitiría hacer descripciones distintas del mismo fenómeno: una neuróloga podría observar desde el exterior (tercera persona) en términos de estructuras y funciones, mientras que nosotros podríamos tener la experiencia fenoménica desde una perspectiva solo describible en primera persona.

Estos principios son altamente debatibles y controversiales. El mismo Chalmers lo reconoce; sin embargo, tiene el mérito de someterlos a la confrontación teórica y empírica.

Su visión emergente fuerte es clara en mi interpretación. No solo las microestructuras y su dinámicas pueden producir efectos macroestructurales y macrodinámicos sino también lo macrodinámico se vuelve irreductible, en tanto propiedad emergente fuerte.

La pregunta que nos podríamos hacer es si la propuesta de Chalmers es, en el fondo, una que permita el avance en la comprensión de la conciencia a partir de la emergencia.

El mayor valor que tiene la propuesta de Chalmers es recordarnos lo 
importante de enfrentar aquellos problemas duros y no simplificarlos como se ha hecho en el pasado. Sin embargo, las respuestas que ofrece a cambio no son convincentes. En primer lugar, es cuestionable que se haga una diferencia tal entre la primera y la tercera persona que, de alguna manera hace inalcanzable la posibilidad de entender el origen de las sensaciones que solemos atribuir a nuestras experiencias fenoménicas. La idea de dividir y vencer no es tan mala en ciertos casos. Concedido que la experiencia fenoménica es el todo que no puede ser lo mismo que las partes neurológicas constituyentes, pero de ahí a estudiar algunas de sus partes para tener alguna intuición que nos permita entender ese difícil fenómeno simplemente no se sigue.

No es el reduccionismo la alternativa para la comprensión de los fenómenos, eso nos ha quedado claro, y con el tiempo, la actitud no-reduccionista se ha convertido en una práctica habitual en la metodología científica -pace los Churchland- ${ }^{4}$ pero, la biología ciertamente nos da lecciones acerca del conocimiento de algunas funciones vitales a partir de las estructuras químicas, como en la Psiquiatría hemos hecho notables avances en la comprensión de los fenómenos psíquicos a partir del estudio de algunos componentes cerebrales, lo que naturalmente no agota la realidad su-

$4 \quad$ P. S. Churchland (1994). perior que supone la conciencia humana. Pero tener la actitud hostil hacia la Biología puede llevar a lo que Dennett (1995) correctamente señala que si no se empieza por dividir los componentes funcionales desde el principio, se puede crear un monstruo, una especie de imaginario resplandor del ojo del homúnculo cartesiano.

Un segundo punto que preocupa en el modelo emergentista de Chalmers es su casi desprecio por la teoría evolucionista como proveedora de explicaciones potenciales para la aparición de la conciencia. En efecto, en sus afirmaciones y demostraciones tanto para la formulación de los principios mencionados como para el desarrollo de la teoría fundamental hace nula referencia a los procesos evolutivos. Pero es una presuposición básica de la Biología que el proceso evolutivo fue responsable del incremento de nuestra capacidad cerebral en el nivel filogenético y la presión del ambiente en el que operaba en el nivel ontogenético. La posición de Chalmers acerca de la evolución se lee en el siguiente pasaje:

Esta idea [la explicación evolutiva de la conciencia] sobreestima lo que una explicación evolutiva puede proporcionarnos. El proceso de selección natural no puede distinguir entre yo y mi gemelo zombi. La evolución selecciona las propiedades de acuerdo con su rol funcional, y mi gemelo zombi realiza todas las funciones que 
yo realizo tan bien como yo; en particular deja alrededor tantas copias de sus genes. Se sigue que la evolución por sí sola no puede explicar porqué las criaturas conscientes en vez de los zombis evolucionaron (1996, p. 120).

Si bien es cierto que ninguna teoría ofrece explicaciones completas, y no tiene por qué hacerlo, los recursos de la teoría evolutiva son mejorables con la investigación y los datos empíricos. El estudio evolutivo de la emergencia del cerebro y de su consecuente conjunto de fenómenos conscientes constituye un enfoque naturalista al problema difícil que Chalmers nos recuerda. La neurofisiología parte del estudio de las estructuras biológicas y funciones que compartimos con otros primates, y todos esos estudios se basan en la teoría evolutiva como marco referencial. Para explicar la superveniencia de lo mental sobre lo físico como ejemplo de la emergencia evolutiva no se puede prescindir de la historia natural que causó que los cerebros y el sistema nervioso en general evolucionen. Prescindir de la teoría, precisamente, no nos permitiría explicar por qué hay seres que experimentan qualia y no zombis que parecen hacerlo. Más difícil sería postular la existencia de zombis dentro del esquema de la teoría de la evolución, porque en general, la teoría no contempla los casos en que los muertos se comporten como vivos.

\section{REFERENCIAS}

Armstrong, D. (1970/1980). The nature of mind. Brisbane: The University of Queensland Press.

Arshinov, V. \& Fuchs, C. (Eds.). (2003). Emergence, causality, selforganisation. Moscú: NIA-Priroda.

Bickerton, D. (2009). Adam's Tongue: How humans made language, how language made humans. Nueva York: Hill and Wang.

Baker, L. R. (1993). Metaphysics and mental causation. En Heil, J. \& Mele, A. (Eds.). Mental Causation. (pp. 75-98). Nueva York: Oxford University Press.

Block, N. (1995). On a confusion about a function of consciousness. Behavioral and Brain Sciences 18, $227-$ 247.

Braun, R. (2009). El rol de la filosofía y la neurociencia. En Quintanilla, P. (Ed.). Ensayos de metafilosofía (pp. 157-178). Lima: Pontificia Universidad Católica del Perú, Fondo Editorial.

Bunge, M. (1977). Levels and reduction. American Journal Regulatory, Integrative and Comparative Physiology, 233 (3), 75-82.

Campbell, D. (1974). La “causación descendente" en los sistemas biológicos jerárquicamente organizados. En Ayala, F. J. \& Dobzhansky, Th. 
(Eds.). Estudios sobre la filosofía de la Biología (pp. 235-245). Barcelona: Ariel.

Chalmers, D. (1995). Facing up the problem of consciousness. Journal of Consciousness Studies, 2, 200-219.

Chalmers, D. (1996). The conscious mind: In search of a fundamental theory. NuevaYork: Oxford University Press.

Chalmers, D. (2006). Strong and weak emergence. En Clayton, P. \& Davies, P. (Eds.). The reemergence of emergence: the emergentist hypothesis from science to religion (pp. 244256). Nueva York: Oxford University Press.

Churchland, P. M. (1981/1991). Eliminative materialism and the propositional attitudes. En Rosenthal, D. (Ed.). The nature of mind. Nueva York: Oxford University Press.

Clayton, P. (2004). Mind and emergence: from quantum to consciousness. Nueva York: Oxford University Press.

Crane, T. (2001). The significance of emergence. En Gillett, C. \& Loewer, B. (Eds.). Physicalism and its Discontents (pp. 207-224). Cambridge: Cambridge University Press.

Dennett, D. (1995). Our vegetative soul-review of Damasio, Descartes' error. Times Literary Supplement, Agosto 25, pp. 3-4.
Emmeche, C., Køppe, S., \& Stjernfelt, F. (1997). Explaining Emergence: Towards an Ontology of Levels. Journal for General Philosophy of Science, 28, 83-117.

Flanagan, O. (1992). Consciousness reconsidered. Cambridge, MA.: The MIT Press.

Harrison, S. (1989). What do viruses look like? Harvey Lectures, 85, 127152. Obtenido de <http://crystal. harvard.edu/lib-sch/HarrisonS 91HLect-85-127.pdf>.

Horgan, T. (1993). From supervenience to superdupervenience: Meeting the demands of a material world. Mind, 10, 555-586.

Humphreys, P. (1997). How properties emerge. Philosophy of Science, 64, $1-17$.

Kim, J. (1993). Supervenience and mind: Selected philosophical essays. Nueva York: Cambridge University Press.

Kim, J. (1999). Making sense of emergence. Philosophical Studies, 95, 3-36.

Kim, J. (2000). Mind in a physical world: An essay on the mind-body problem and mental causation. Cambridge, MA.: The MIT Press.

Levine, J. (2001). Purple haze: The puzzle of consciousness. Nueva York: Oxford University Press. 
Locke, J. (1690/2008). An essay concerning human understanding. Nueva York: Oxford University Press.

Nagel, T. (1974). What is it like to be a bat? Philosophical Review, 83, 7-22.

Nagel, T. (1985). The view from nowhere. Nueva York: Oxford University Press.

Putnam, H. (1980). The nature of mental states. En Block, N. (Ed.). Readings in the philosophy of psyhology. Vol. 1. Cambridge, MA.: Harvard University Press.

Reid, R. (2007). Biological emergences: Evolution by natural experi- ment. Cambridge, MA.: The MIT Press.

Robinson, W. (2007). Epiphenomenalism (2007). En Stanford Encyclopedia of Philosophy. Obtenido de http://plato.stanford.edu/entries/epiphenomenalism/

Smart, J. J. C. (1962/1991). Sensations and brain processes. En Rosenthal, $\mathrm{R}$. (Ed.). The nature of mind. Nueva York: Oxford University Press.

Stephan, A. (1999). Varieties of emergentism. Evolution and Cognition, 5, 49-59. 